\title{
Gurinder Singh Grewal: can the new medical council president clean up medicine in Punjab?
}

In this Feature (BMJ 2015;351:h3694, doi:10.1136/bmj.h3694) we incorrectly stated that the suspected ghost doctors had been given court orders, when they were actually issued with "show 